\title{
Euclid: Forecast constraints on the cosmic distance duality relation with complementary external probes ${ }^{\star}$
}

M. Martinelli ${ }^{1}$, C. J. A. P. Martins ${ }^{2,3}$, S. Nesseris ${ }^{1}$, D. Sapone ${ }^{4}$, I. Tutusaus ${ }^{5,6}$, A. Avgoustidis ${ }^{7}$, S. Camera ${ }^{8,9,10}$, C. Carbone ${ }^{11}$, S. Casas ${ }^{12}$, S. Ilić ${ }^{13,14,15}$, Z. Sakr ${ }^{15,16}$, V. Yankelevich ${ }^{17}$, N. Auricchio ${ }^{18}$, A. Balestra ${ }^{19}$, C. Bodendorf ${ }^{20}$, D. Bonino ${ }^{10}$, E. Branchini ${ }^{21,22,23}$, M. Brescia ${ }^{24}$, J. Brinchmann ${ }^{3}$, V. Capobianco ${ }^{10}$, J. Carretero ${ }^{25}$, M. Castellano ${ }^{23}$, S. Cavuoti ${ }^{24,26,27}$, R. Cledassou ${ }^{28}$, G. Congedo ${ }^{29}$, L. Conversi $^{30,31}$, L. Corcione $^{10}$, F. Dubath ${ }^{32}$, A. Ealet $^{33}$, M. Frailis ${ }^{34}$, E. Franceschi ${ }^{18}$, M. Fumana ${ }^{11}$, B. Garilli ${ }^{11}$, B. Gillis ${ }^{29}$, C. Giocoli ${ }^{35,36,37}$, F. Grupp ${ }^{20,38}$, S. V. H. Haugan ${ }^{39}$, W. Holmes ${ }^{40}$, F. Hormuth ${ }^{41}$, K. Jahnke ${ }^{42}$, S. Kermiche ${ }^{43}$, M. Kilbinger ${ }^{12}$, T. D. Kitching ${ }^{44}$,

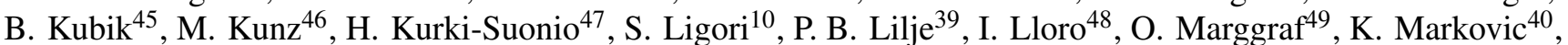
R. Massey ${ }^{50}$, S. Mei ${ }^{51,52}$, M. Meneghetti ${ }^{35,37}$, G. Meylan ${ }^{53}$, L. Moscardini ${ }^{18,36,54}$, S. Niemi ${ }^{44}$, C. Padilla ${ }^{55}$, S. Paltani ${ }^{32}$, F. Pasian ${ }^{34}$, V. Pettorino ${ }^{12}$, S. Pires ${ }^{12}$, G. Polenta ${ }^{56}$, M. Poncet ${ }^{28}$, L. Popa ${ }^{57}$, L. Pozzetti ${ }^{18}$, F. Raison ${ }^{20}$, J. Rhodes ${ }^{40}$, M. Roncarelli ${ }^{18,36}$, R. Saglia ${ }^{20,38}$, P. Schneider ${ }^{49}$, A. Secroun ${ }^{43}$, S. Serrano ${ }^{5,6}$, C. Sirignano ${ }^{58,59}$,

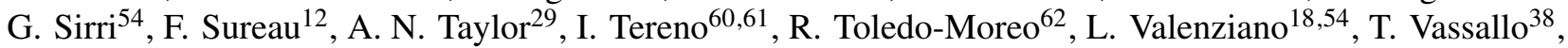
Y. Wang ${ }^{63}$, N. Welikala ${ }^{29}$, J. Weller ${ }^{20,38}$, and A. Zacchei ${ }^{34}$

(Affiliations can be found after the references)

Received 31 July 2020 / Accepted 5 October 2020

\begin{abstract}
Context. In metric theories of gravity with photon number conservation, the luminosity and angular diameter distances are related via the Etherington relation, also known as the distance duality relation (DDR). A violation of this relation would rule out the standard cosmological paradigm and point to the presence of new physics.

Aims. We quantify the ability of Euclid, in combination with contemporary surveys, to improve the current constraints on deviations from the DDR in the redshift range $0<z<1.6$.

Methods. We start with an analysis of the latest available data, improving previously reported constraints by a factor of 2.5 . We then present a detailed analysis of simulated Euclid and external data products, using both standard parametric methods (relying on phenomenological descriptions of possible DDR violations) and a machine learning reconstruction using genetic algorithms.

Results. We find that for parametric methods Euclid can (in combination with external probes) improve current constraints by approximately a factor of six, while for non-parametric methods Euclid can improve current constraints by a factor of three.

Conclusions. Our results highlight the importance of surveys like Euclid in accurately testing the pillars of the current cosmological paradigm and constraining physics beyond the standard cosmological model.
\end{abstract}

Key words. cosmology: observations - cosmological parameters - surveys - methods: data analysis - methods: statistical space vehicles: instruments

\section{Introduction}

Standard cosmological analyses rely on several explicit or implicit assumptions. Three examples of commonly made assumptions are that the Copernican principle holds (i.e. we are not at a special place in the Universe), that the photon number is conserved, and that the Universe is homogeneous and isotropic, at least on sufficiently large scales. While they are, in some sense, the pillars on which standard cosmology is built and none of them are seriously challenged by current data (for a possible exception see Webb et al. 2011), they are violated in many extensions of the standard cosmological model and of the standard particle physics paradigm, with extensions of the latter collectively known as beyond the standard model (BSM) theories. In this work, we focus on another of these assumptions, which is related to, but conceptually different from, the previous three: the so-called distance duality relation (DDR), which

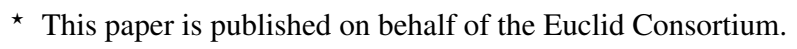

is crucial for tests of the background expansion rate of the Universe as it allows us to relate the luminosity and angular diameter distances while at the same time affecting the prediction for the change in redshift of the cosmic microwave background (CMB) radiation temperature. Specifically, the DDR holds true for metric theories of gravity where the photon number is conserved and photons travel along null geodesics. Testing these predictions with cosmological data therefore has the potential to rule out large classes of extended theories or to observe signatures of non-standard physics.

Combining supernova data from the supernova cosmology project (SCP) Union 2008 compilation (Kowalski et al. 2008) and $H(z)$ data from Stern et al. (2010), deviations from the standard DDR have been constrained to few percent (Avgoustidis et al. 2010; Ma \& Corasaniti 2018). At the same time, direct measurements of CMB temperature at different redshifts, denoted $T(z)$ hereafter, have been obtained at both low redshifts $(z \lesssim 1)$ via observations of the Sunyaev-Zel'dovich effect in galaxy clusters (Luzzi et al. 2009) and at higher 
redshifts $(z>1)$ through high-resolution spectroscopy of atomic, ionic, or molecular levels excited by the absorption of CMB photons (Noterdaeme et al. 2011). The deviation from the standard redshift evolution of CMB temperature has also been constrained to a few percent (Noterdaeme et al. 2011). In models where deviations from the DDR arise due to an effective opacity effect (e.g. dimming due to photons decaying into an unobserved particle), the same physical mechanism can also give rise to a related violation of the standard $T(z)$ evolution. For such models, the combination of distance and temperature measurements as two independent probes of the same underlying mechanism shrunk the constraints on the deviation of $T(z)$ to $0.8 \%$ (Avgoustidis et al. 2016). Other possible observables to constrain possible violations of the DDR include galaxy clusters (Holanda et al. 2010; Li et al. 2011), the Sunyaev-Zeldovich effect (Holanda et al. 2012), strong gravitational lensing (Liao et al. 2016), and standard sirens from gravitational wave observations (Liao 2019; Hogg et al. 2020).

Upcoming and more sensitive cosmological surveys for supernovae and baryon acoustic oscillations (BAO) will further tighten the constraints achievable through distance measurements. Here we focus on Euclid, an M-class space mission of the European Space Agency due for launch in 2022. It will carry two different instruments on board: a visible imager (Cropper et al. 2018) and a near-infrared spectrophotometric instrument (Costille et al. 2018). Together they will carry out a photometric and spectroscopic galaxy survey over $15000 \mathrm{deg}^{2}$ of extra-galactic sky with the aim of measuirng the geometry of the Universe and the growth of structures up to $z \sim 2$ and beyond (Laureijs et al. 2011).

Euclid will have three main cosmological probes: weak lensing and galaxy clustering from the photometric survey and galaxy clustering from the spectroscopic survey. While photometric galaxy surveys allow for observations of large numbers of galaxies with relatively large redshift uncertainties, spectroscopic galaxy surveys provide information for fewer objects but with much higher radial precision. The spectroscopic accuracy of Euclid will allow for precise galaxy clustering analyses that include the radial dimension. Here we simulate BAO data from Euclid using the Fisher matrix technique and specifically following the same strategy used in Euclid Collaboration (2020a), hereafter EC19, for the spectroscopic survey.

On the other hand, improved direct measurements of the CMB temperature at different redshifts will be available, such as those expected in the coming years from ESPRESSO (Pepe et al. 2013; Leite et al. 2016), and eventually ELT-HIRES (Liske 2014), and these will significantly improve the available constraining power (Avgoustidis et al. 2014). In addition to this, future observations of gravitational wave events will allow us to exploit standard sirens to obtain luminosity distance measurements at even higher redshifts, thus extending the redshift range of DDR tests (see for example Yang et al. 2019).

Our work also highlights some of the synergies between different surveys. Specifically, we will show how data from the Dark Energy Spectroscopic Instrument (DESI, DESI Collaboration 2016), a survey that aims at probing the expansion rate and large-scale structure (LSS) of the universe, and from the Legacy Survey of Space and Time (LSST), performed by the Vera C. Rubin Observatory (Abell 2009), can complement the Euclid $\mathrm{BAO}$ survey and extend the probed redshift range.

In Amendola et al. (2018), forecast constraints on deviations from the standard DDR were presented (and their implications on mechanisms of cosmic opacity explored), combining a dark energy task force stage IV supernova mission (Albrecht et al.
2006, taking SNAP as a concrete example) and the galaxy survey expected to be performed by the Euclid satellite (Laureijs et al. 2011). In this paper, we aim to update and extend those results on constraining DDR deviations; specifically, we rely on more recent Euclid specifications (see EC19) and we investigate possible synergies between this survey and contemporary observations. We also refine the analysis done in Amendola et al. (2018); we follow the common approach of encoding DDR violations in the phenomenological function $\epsilon(z)$, but, alongside the common constant parameterization, we include here a binning in redshift of this function in order to understand if current or future data are able to detect a redshift trend. Moreover, we also apply a more refined machine learning technique to reconstruct $\epsilon(z)$ with a minimal set of assumptions, performing our analysis with the use of genetic algorithms (GAs) (Bogdanos \& Nesseris 2009; Nesseris \& Garcia-Bellido 2012).

After reviewing the theoretical background of the DDR in Sect. 2, we describe the different analyses done in this work to test the relation in Sect. 3, detailing both our parameterized approach and the agnostic reconstruction. We then present the constraints obtained from current observations in Sect. 4. The results of this analysis are used as a fiducial cosmology for the mock data we produce in Sect. 5 and then compared with forecast results, which are discussed in Sect. 6. Finally, we draw our conclusions in Sect. 7.

\section{Extensions of the distance duality relation}

In any cosmological model based on a metric theory of gravity, the Etherington relation (Etherington 1933), also known as the DDR, implies that distance measures are unique. The luminosity distance, $d_{\mathrm{L}}(z)$, is related to the angular diameter distance, $d_{\mathrm{A}}(z)$, as

$d_{\mathrm{L}}(z)=(1+z)^{2} d_{\mathrm{A}}(z)$

and this relation is valid in any cosmological background where photons travel on null geodesics and where, crucially, the photon number is conserved. Deviations from DDR as expressed in Eq. (1) can be directly constrained by data (for example using a phenomenological parameterization), but one is often interested in constraining the underlying physical mechanism giving rise to such deviations within a class of cosmological models. For example, in models where photons can decay into another particle that remains unobserved, there is an effective violation of photon number conservation which can be used to constrain the coupling between the photon and the new particle.

On the other hand, if the expansion of the Universe is adiabatic and the CMB spectrum was a black-body at the time it originated, such a property will be preserved by the subsequent cosmological evolution, with the CMB temperature evolving as

$T(z)=T_{0}(1+z)$.

This is a robust prediction of standard cosmology, but it is violated in many non-standard models, including scenarios involving photon mixing (for a review see Jaeckel \& Ringwald 2010) and the violation of photon number conservation, which may also induce deviations from the DDR. Throughout this paper we assume that the cosmological principle holds, namely that the Universe is, to first approximation, homogeneous and isotropic. Consequently, we do not investigate any possible dependence of the DDR on the direction of the sky and only tackle its possible redshift dependence (for further discussion on the validity of 
such an assumption see for example Maartens 2011; Ntelis et al. 2017).

From a theoretical point of view, models violating the DDR through a violation of photon number conservation are of particular interest and, as has been alluded to above, in such extended theories one expects deviations from both Eqs. (1) and (2). Therefore, an analysis of such behaviour could in principle benefit from the complementarity between galaxy and supernova surveys, probing departures from the DDR, and spectroscopic tests of $T(z)$ evolution, both of which will be available during the next decade.

Deviations from the Etherington relation are commonly parameterized as

$d_{\mathrm{L}}(z)=(1+z)^{2+\epsilon(z)} d_{\mathrm{A}}(z)$,

where the function $\epsilon(z)$ is usually assumed to be constant and, using currently available data, its value is constrained to be $O\left(10^{-2}\right)$ (Avgoustidis et al. 2009, 2010). As the precision of the data improves and the available redshift range is extended, the DDR could be probed at larger redshifts, $z \gtrsim 2$, and tighter constraints of a possible redshift dependence could, in principle, be obtained. Deviations from the standard DDR are also commonly encoded in a function $\eta(z)$, defined as

$\eta(z)=\frac{d_{\mathrm{L}}(z)}{d_{\mathrm{A}}(z)(1+z)^{2}}=(1+z)^{\epsilon(z)}$.

Similarly, deviations from the standard evolution of the CMB temperature with redshift can be parametrized phenomenologically by (Lima et al. 2000; Luzzi et al. 2009; Avgoustidis et al. 2012)

$T(z)=T_{0}(1+z)^{1-\beta}$,

where for simplicity it is assumed that violations of the standard behaviour are achromatic (they do not depend on the photons' wavelength) and approximately adiabatic, so the spectrum of CMB radiation remains approximately a black body spectrum. A discussion of these assumptions can be found in Avgoustidis et al. (2016). It is important to stress that in a broad range of models where the photon number is not conserved, and so the temperature-redshift relation and the DDR are both violated, the functions parameterizing these two possible violations will not be independent. Defining a generic function $f(z)$ encoding the violation of the temperature-redshift relation as

$T(z)=T_{0}(1+z) f(z)$,

it is possible to show (Avgoustidis et al. 2012) that the DDR violation will then be

$d_{\mathrm{L}}(z)=d_{\mathrm{A}}(z)(1+z)^{2} f(z)^{3 / 2}$.

Therefore, for the two simple parameterizations introduced above, the parameterized deviations from the standard model are related as

$\epsilon=-\frac{3}{2} \beta$.

In this work we focus mainly on constraining violations from the DDR using supernova and BAO data, but have in mind a specific class of physical mechanisms producing violations of both Eqs. (1) and (2), due to a change in the photon flux during the propagation from distant sources. Such mechanisms would affect the supernova luminosity distance measures but not the determinations of the angular diameter distance. This means that probes of the latter (BAO) can be combined with supernova surveys to constrain deviations from photon number conservation. In addition, it also means that direct measurements of the CMB temperature at different redshifts could be used to further improve the available constraining power, as mentioned above.

Photon conservation can be violated by simple astrophysical effects or by exotic physics. Amongst the former we find, for instance, attenuation due to interstellar dust, gas, and/or plasma. Such astrophysical mechanisms produce an effective opacity, which would correspond to a positive value of the phenomenological parameter $\epsilon$. Most known sources of attenuation are expected to be clustered and can be typically constrained down to the $0.1 \%$ level (Ménard et al. 2008; More et al. 2009).

Unclustered sources of attenuation are more difficult to constrain. Grey dust (Aguirre 1999) was initially invoked to explain the observed dimming of Type Ia supernovae (SnIa) without resorting to cosmic acceleration. While this has been subsequently ruled out by observations (Aguirre \& Haiman 2000; Bassett \& Kunz 2004), it has been shown (Corasaniti 2006) that the effect of grey dust could cause an extinction as large as $0.08 \mathrm{mag}$ at $z=1.7$, thus potentially affecting dark energy parameter inference from future supernova surveys.

Concerning exotic physics explanations, a possible source of photon conservation violation is the coupling of photons with particles beyond the standard model of particle physics. Such couplings would mean that, while passing through the intergalactic medium, a photon could disappear, or even (re)appear, while interacting with such exotic particles, modifying the apparent luminosity of sources. Therefore, in this case, we may in principle envisage both positive and negative values for $\epsilon$. In Avgoustidis et al. (2010), the mixing of photons with several such particles is considered and constrained in three representative scenarios: scalars known as axion-like particles (Svrcek \& Witten 2006), chameleons (Brax et al. 2010), and the possibility of mini-charged particles, which have a tiny and unquantized electric charge (Holdom 1986; Batell \& Gherghetta 2006). The implications of each of these three specific scenarios for the SnIa luminosity have been described by several authors (Csáki et al. 2002; Mörtsell et al. 2002; Burrage 2008; Ahlers 2009).

Finally, it is worth noting that any violations in photon conservation can be described as an opacity effect in the observed luminosity distance, which one can parameterize through a generic opacity parameter, $\tau(z)$, as

$d_{\mathrm{L}, \mathrm{obs}}^{2}=d_{\mathrm{L}, \text { true }}^{2} \exp [\tau(z)]$.

We note that a negative $\tau(z)$ allows for apparent brightening of light sources, as would be the case, for example, if exotic particles were also emitted from the source and converted into photons along the line of sight (see Burrage 2008). For specific models of exotic matter-photon coupling, such as axion-like particles, chameleons, and mini-charged particles, the function $\tau(z)$ can be obtained in terms of the parameters of the model (Avgoustidis et al. 2010).

\section{Analysis method}

In this paper, we aim at obtaining constraints on possible deviations from the standard DDR, without assuming any specific model, from both current and mock data. For this reason we adopt two different approaches: on the one hand we parameterize the $\epsilon(z)$ function, both as a constant and binning it in redshift, while on the other hand we also adopt a more general approach based on machine learning, reconstructing the function with GAs. In this section we review in detail the two approaches. 


\subsection{Parameterized approach}

A first simple way to constrain the cosmic DDR is to parameterize departures from the Etherington relation through a constant (redshift-independent) parameter $\epsilon_{0}$, that is

$d_{\mathrm{L}}(z)=(1+z)^{2+\epsilon_{0}} d_{\mathrm{A}}(z)$,

with $\epsilon_{0}=0$ being the standard limit. However, we are also interested in a possible redshift dependence of such departures, as many of the theoretical models discussed in Sect. 2 produce a redshift dependent modification of the DDR. Therefore, we take one step further by using the general form of Eq. (3). Choosing a specific model violating the Etherington relation would allow us to obtain $\epsilon(z)$ in terms of the parameters of the chosen model. However, the aim of this paper is not to constrain specific theories; in order not to make strong assumptions on the redshift dependence of $\epsilon(z)$, one could exploit parameterizations of the redshift trend of such a function (Lv \& Xia 2016). Instead, we consider a simple binning of this function in two redshift bins, that is

$\epsilon(z)= \begin{cases}\epsilon_{0} & \text { if } z<z_{*}, \\ \epsilon_{1} & \text { if } z \geq z_{*},\end{cases}$

where $z_{*}$ is a transition redshift; we will comment on the choice of this redshift in the results section.

In order to constrain these two parameterizations, we implement them in a new likelihood module interfaced with the publicly available MCMC sampler Cobaya (Torrado \& Lewis 2020), able to reconstruct the posterior distribution of cosmological parameters, using SnIa and BAO data coming from current surveys or from simulated datasets. SnIa data are compared with the theoretical predictions given by Eq. (3), while with BAO data we compare combinations of the Hubble parameter $H(z)$ and of the standard angular diameter distance of Eq. (12).

We assume for this parameterized approach that the Universe expansion is well described by a flat $\Lambda C D M$ model, with the late time evolution dominated by a cosmological constant with equation of state parameter $w(z)=-1$. Given the flatness assumption, the angular diameter distance appearing in Eq. (10) can be obtained in terms of the Hubble parameter $H(z)$ as

$d_{\mathrm{A}}(z)=\frac{c}{1+z} \int_{0}^{z} \frac{\mathrm{d} z^{\prime}}{H\left(z^{\prime}\right)}$.

Therefore, we sample through Cobaya $\epsilon_{0}$ and $\epsilon_{1}$, parameterizing deviations from the standard DDR, alongside the total energy density of matter $\Omega_{\mathrm{m}, 0}$, and the Hubble constant $H_{0}$, using flat priors on these parameters. The assumption of a flat Universe implies that the energy density given by the cosmological constant $\Lambda$ is $\Omega_{\Lambda, 0}=1-\Omega_{\mathrm{m}, 0}$, neglecting the contribution of radiation energy density since we are analysing low-redshift data. Furthermore, we fix the baryon energy density to the mean obtained by Planck $\Omega_{\mathrm{b}, 0} h^{2}=0.02225$ (Planck Collaboration VI 2020). Abandoning the assumption of $\Lambda \mathrm{CDM}$ and allowing for free parameters describing the equation of state of the new dark energy component would impact the constraints on DDR violation parameters, with the possibility of introducing degeneracies between the parameters determining $w(z)$ and $\epsilon(z)$; we leave however the investigation of this possibility for future work.

\subsection{Genetic algorithms}

The GAs represent a class of machine learning methods that can be used for non-parametric reconstruction of data and are based on the notions of grammatical evolution, as expressed by the genetic operations of crossover and mutation. In particular, the GAs mimic the principle of evolution through the implementation of natural selection; a group of individuals evolves over time under the influence of the stochastic operators of mutation, namely a random change in an individual, and crossover, that is the combination of different individuals to form offspring.

The probability that a member of the population will produce offspring, or in other terms its "reproductive success", is assumed to be proportional to its fitness. The latter measures how accurately each individual of the population fits the data, here quantified through a $\chi^{2}$ statistic (for more details on the GA and various applications to cosmology see Bogdanos \& Nesseris 2009; Akrami et al. 2010; Nesseris \& Shafieloo 2010; Nesseris \& Garcia-Bellido 2012, 2013; Sapone et al. 2014; Arjona \& Nesseris 2020a,b; Arjona 2020), which is obtained following the same likelihood computation used in Sect. 3.1.

Qualitatively, the joint reconstruction of the SnIa and BAO data with the GA proceeds as follows. An initial population of functions is randomly chosen such that every member of the population contains initial guesses for both the luminosity distance $d_{\mathrm{L}}(z)$ and the duality parameter $\eta(z)$. At this point we also impose some physical priors, such as that the luminosity distance at $z=0$ is zero, but we make no assumption on a DE model. Then, each member's fitness is calculated via a $\chi^{2}$ statistic, using as input the SnIa and BAO data and their individual covariances. Subsequently, the mutation and crossover operators are applied to the best-fitting functions in every generation, chosen via tournament selection-see Bogdanos \& Nesseris (2009) for more details. This process is then iterated thousands of times, so as to ensure convergence, and with different random seeds, so as not to bias the results due to a specific choice of the random seed.

After the GAs code has converged, the final output is a pair of two continuous and differentiable functions of redshift that describe the luminosity distance $d_{\mathrm{L}}(z)$ and the duality parameter $\eta(z)$, respectively. At every step the angular diameter distance is calculated following Eq. (4), while the Hubble parameter $H(z)$ is calculated via differentiation of the latter assuming flatness. In the case of the current data we also numerically minimize the $\chi^{2}$ at every step over the combination $r_{\mathrm{s}}\left(z_{\mathrm{d}}\right) h$, with $r_{\mathrm{s}}\left(z_{\mathrm{d}}\right)$ the comoving sound horizon at the drag epoch and $h=H_{0} /\left(100 \mathrm{~km} \mathrm{~s}^{-1} \mathrm{Mpc}^{-1}\right)$, in order to avoid making any model assumptions for the BAO physics at early times.

To estimate the errors on the reconstructed functions, we use an analytical approach developed by Nesseris \& Garcia-Bellido (2012, 2013), where the errors are calculated via a path integral over the whole functional space that can be scanned by the GA. The GA path integral approach was extensively tested by Nesseris \& Garcia-Bellido (2012) and found to be in excellent agreement with bootstrap Monte-Carlo error estimates.

In summary, using this approach we can reconstruct any cosmological function, for example the luminosity distance $d_{\mathrm{L}}(z)$ or the duality parameter $\eta(z)$ that we consider here, by applying the GA to any dataset of choice. No assumptions on the specific cosmological model or the behaviour of DE need to be made, hence our results are independent from specific DDR violation models. Since in our case the best-fit is very close to $\Lambda \mathrm{CDM}$ and the errors are much larger than the effects of any possible modelbias in the covariances of the data, we can safely assume for the time being that these effects have a rather minimal impact on the whole minimization process.

Finally, for the numerical implementation of the GA used in this paper we use the publicly available code Genetic 
Algorithms ${ }^{1}$. In addition to performing a large number of GA runs with different random seed numbers, we have also required that all reconstructed functions, as well as their derivatives, are continuous in the range of redshifts we consider, in order to avoid spurious reconstructions and overfitting.

\section{Analysis of currently available data}

In order to constrain the deviation from the standard DDR, we need to analyse a set of data providing information on the luminosity and angular diameter distances. We focus therefore on currently available observations of SnIa and BAO.

The BAO data will provide information on the angular diameter distance $d_{\mathrm{A}}(z)$ and the Hubble parameter $H(z)$. We use here measurements of the ratio $d_{z}$, defined as

$d_{z} \equiv \frac{r_{\mathrm{s}}\left(z_{\mathrm{d}}\right)}{D_{V}(z)}$

where $D_{V}$ is the volume averaged distance

$D_{V}(z)=\left[(1+z)^{2} d_{\mathrm{A}}^{2}(z) \frac{c z}{H(z)}\right]^{1 / 3}$,

and $r_{\mathrm{s}}\left(z_{\mathrm{d}}\right)$ is the comoving sound horizon at the drag epoch

$r_{\mathrm{s}}\left(z_{\mathrm{d}}\right)=\frac{1}{H_{0}} \int_{z_{\mathrm{d}}}^{\infty} \frac{c_{\mathrm{s}}(z)}{H(z) / H_{0}} \mathrm{~d} z$,

with $c_{\mathrm{S}}(z)$ the sound speed and $z_{\mathrm{d}}$ the redshift at the drag epoch (see Eq. (4) of Eisenstein \& Hu 1998). In the $\Lambda$ CDM model, Eq. (15) can be approximated as (see Eq. (26) of Eisenstein \& $\mathrm{Hu}$ 1998)

$r_{\mathrm{s}}\left(z_{\mathrm{d}}\right) \simeq \frac{44.5 \log \left(\frac{9.83}{\Omega_{\mathrm{m}, 0} h^{2}}\right)}{\sqrt{1+10\left(\Omega_{\mathrm{b}, 0} h^{2}\right)^{3 / 4}}} \mathrm{Mpc}$.

Throughout this paper we will assume that this approximation holds in all our parameterized analyses. Moreover, as the data combination considered here cannot constrain $\Omega_{\mathrm{b}, 0} h^{2}$, we assume the value $\Omega_{\mathrm{b}, 0} h^{2}=0.02225$ from Planck 2018 (Planck Collaboration VI 2020). We notice that the constraints one can obtain through analysis of the BAO can depend significantly on this assumption, with different choices available on how to obtain prior information on $r_{\mathrm{s}}\left(z_{\mathrm{d}}\right)$ (see for example Cuesta et al. 2015 , for a detailed discussion on the role of $r_{\mathrm{s}}\left(z_{\mathrm{d}}\right)$ assumptions in BAO analysis). For instance, a change of $1 \%$ in the value of $\Omega_{\mathrm{b}, 0} h^{2}$ leads to a change of about $2 \%$ on the distance ratio $d_{z}$. The observational constraints, on the other hand, on the quantity given by Eq. (13), as well as on the Hubble distance $D_{\mathrm{H}}(z)=c / H(z)$, that we consider here are provided by the surveys 6dFGS (Beutler et al. 2011), SDDS (Anderson et al. 2014), BOSS CMASS (Xu et al. 2012), WiggleZ (Blake et al. 2012), MGS (Ross et al. 2015), BOSS DR12 (Gil-Marín et al. 2016), DES (Abbott et al. 2019), Ly- $\alpha$ observations from Blomqvist et al. (2019), SDSS DR14 LRG (Bautista et al. 2018) and quasars observations from Ata et al. (2018). In the rest of the paper, we will refer to the combination of these datasets as BAO, for simplicity. We refer the reader to Appendix A for further details on how these datasets are combined together and a description of their likelihood.

We also note that the BAO data may have some model dependence, as a fiducial cosmology is required in order to convert the

\footnotetext{
1 https://github.com/snesseris/Genetic-Algorithms
}

measured angular scales to distances, while some more uncertainty may also be introduced by the non-linear effects which damp and modify the position of the BAO in the galaxy power spectrum. Both of these issues of course imply that systematic errors of a few percent may be introduced in the inferred cosmological parameters (see Angulo et al. 2008). While it is possible to standardise the BAO distance measurements, many of these techniques are based on the particular modelling of nonlinear scales, something which is quite complicated for theories beyond the $\Lambda \mathrm{CDM}$ model. Not including the non-linear modelling, may thus lead to reduced constraining power (see for example Anselmi et al. 2018).

On the other hand, the SnIa data provide information on the luminosity distance $d_{\mathrm{L}}(z)$, as the measured observable is the apparent magnitude $m(z)$ which can be expressed as

$m(z)=M_{0}+5 \log _{10}\left(\frac{d_{\mathrm{L}}(z)}{\mathrm{Mpc}}\right)+25$,

where $M_{0}$ is the intrinsic magnitude of the considered supernova. Such a quantity is completely degenerate with the Hubble constant $H_{0}$, thus, if no external information is provided, SnIa data are not able to constrain these two quantities. Here, we analyse the SnIa data using the likelihood expression from Appendix C in Conley et al. (2011), which already takes into account the marginalization of $M_{0}$ and $H_{0}$ from the SnIa analysis. The dataset we consider for the SnIa is the updated Pantheon compilation of 1048 points from Scolnic et al. (2018).

\subsection{Parameterized results}

Using the surveys described above we can quantify the current constraining power on the DDR, both in the constant and binned cases of the $\epsilon$ parameterization. In Table 1 we report both of these results, whereas in Fig. 1 we show the constraints obtained from the considered observables, both separately and in combination. The contours shown here clarify how SnIa data are able to constrain $\epsilon(z)$, although the degeneracy between $\Omega_{\mathrm{m}, 0}$ and the DDR parameters significantly limits the constraining power. The BAO data on the other hand, are not sensitive to $\epsilon(z)$, but are able to obtain tight constraints on the allowed matter density. Therefore, when the two datasets are combined the degeneracy between $\Omega_{\mathrm{m}, 0}$ and $\epsilon_{0}, \epsilon_{1}$ is broken.

One may notice that the mean of the DDR parameters is positive, both in the constant and binned cases, and that the inclusion of BAO data shifts the constraints towards the standard cosmology limit $\epsilon(z)=0$. Using alternative BAO combinations, producing different constraints on $\Omega_{\mathrm{m}, 0}$, can therefore lead to different results on $\epsilon(z)$; this is crucial if one wants to connect the constraints to viable theoretical models producing the inferred violation of DDR, as the mechanisms leading to a positive or negative $\epsilon(z)$ can be significantly different, as discussed in Sect. 2.

Considering the combined Pantheon $+\mathrm{BAO}$ constraints, in the constant $\epsilon(z)$ case, the posterior distribution peaks at $\epsilon_{0} \neq 0$; however, within the $1 \sigma$ limit, the result is compatible with zero. The binned $\epsilon(z)$ case shows a similar behaviour: Both the first and second bin parameters, that is $\epsilon_{0}$ and $\epsilon_{1}$, are compatible with zero at $1 \sigma$. Furthermore, the errors on these two parameters are very similar, showing how current data provide similar constraining power in the two redshift bins considered here. This is due to the fact that the transition redshift $z_{*}$, fixed here to $z_{*}=0.9$, lies roughly midway through the redshift range of the SnIa data, which are those sensitive to DDR parameters. It is important to stress here that throughout this analysis, we keep 
Table 1. Mean values and marginalized $68 \%$ confidence level errors obtained from currently available data on the cosmological parameters $\Omega_{\mathrm{m}, 0}$ and $H_{0}$ (in units of $\mathrm{km} \mathrm{s}^{-1} \mathrm{Mpc}^{-1}$ ) and on the DDR parameters $\epsilon_{0}$ and $\epsilon_{1}$ (if present).

\begin{tabular}{lccc}
\hline \hline & & Constant $\epsilon(z)$ & Binned $\epsilon(z)$ \\
\hline Parameter & Probe & & \\
\hline \multirow{4}{*}{$H_{0}$} & BAO & $66.6_{-1.4}^{+1.3}$ & $66.7 \pm 1.3$ \\
& SnIa & Unconstrained & Unconstrained \\
& SnIa+BAO & $66.6 \pm 1.3$ & $66.6 \pm 1.3$ \\
\hline \multirow{4}{*}{$\Omega_{\mathrm{m}, 0}$} & BAO & $0.300_{-0.036}^{+0.027}$ & $0.302_{-0.035}^{+0.027}$ \\
& SnIa & $0.329_{-0.12}^{+0.094}$ & $0.357_{-0.14}^{+0.090}$ \\
& SnIa+BAO & $0.301_{-0.034}^{+0.028}$ & $0.301_{-0.033}^{+0.026}$ \\
$\epsilon_{0}$ & BAO & Unconstrained & Unconstrained \\
& SnIa & $0.030 \pm 0.088$ & $0.056_{-0.10}^{+0.087}$ \\
& SnIa+BAO & $0.013 \pm 0.029$ & $0.015_{-0.031}^{+0.027}$ \\
\hline \multirow{4}{*}{$\epsilon_{1}$} & BAO & - & Unconstrained \\
& SnIa & - & $0.046 \pm 0.089$ \\
& SnIa+BAO & - & $0.009 \pm 0.030$ \\
\hline
\end{tabular}

the transition redshift $z_{*}$ fixed. Moreover, current data do not provide any hint for $\epsilon_{0} \neq \epsilon_{1}$, as the constraints of both parameters are compatible with each other and therefore consistent with a constant $\epsilon(z)$. We analysed the data also allowing for a free $z_{*}$; however, as the data are compatible with a constant $\epsilon(z)$, no clear peak of the posterior distribution is present, with the two extreme cases $z_{*} \approx 0$ and $z_{*} \gtrsim 2$ providing the same result. Therefore, the posterior distribution is extremely difficult to sample with the MCMC algorithm used here and we decide to present here only the analysis where $z_{*}$ is fixed.

In particular, using both the current SnIa and BAO data, we find $\epsilon_{0}=0.013 \pm 0.029$, while, when the binned approach is used, we find $\epsilon_{1}=0.009 \pm 0.030$ and $\epsilon_{0}=0.015_{-0.031}^{+0.027}$. The former may be compared to the analysis by Avgoustidis et al. (2010), where $\epsilon_{0}$ was found to be $\epsilon_{0}=-0.04_{-0.07}^{+0.08}$ (all of these being at the $68 \%$ confidence level).

Finally, we show in Fig. 2 the reconstructed trend of $\eta(z)$, whose values at different redshifts are obtained as a derived parameter using Eq. (4). As can be seen, the reconstruction is in agreement with $\Lambda \mathrm{CDM}$ within the errors.

\subsection{GA sresults}

In order to obtain constraints on the violation of the DDR without assuming any specific trend in redshift for $\epsilon(z)$, we employ here the GAs approach described in Sect. 3.2, applied simultaneously to the currently available SnIa and BAO data. We find that a joint fit with the GAs to both data sets gives a competitive fit with respect to the $\Lambda \mathrm{CDM}$ model. In particular, after applying the GAs we find a best-fit of $\chi_{\min , \mathrm{GA}}^{2}=1041.510$ for $r_{\mathrm{s}}\left(z_{\mathrm{d}}\right)=$ $100.360 \mathrm{Mpc} / h$. Concerning the $\Lambda \mathrm{CDM}$ model, we obtain a minimum value of $\chi_{\min , \Lambda \mathrm{CDM}}^{2}=1045.696$, for $1048+12=1060$ data points (1048 from the SnIa and 12 from the BAO), for the best-fit matter density parameter $\Omega_{\mathrm{m}, 0}=0.297 \pm 0.018$ and $H_{0}=66.7 \pm 1.0 \mathrm{~km}^{-1} \mathrm{~s}^{-1} \mathrm{Mpc}^{-1}$. Overall, the GA provides a better fit to the data, with $\Delta \chi^{2}=4.187$, compared to the $\Lambda \mathrm{CDM}$ model.

As mentioned before, the output of the GAs is an analytical function, but in most cases the exact expression is both cumbersome and not informative. Though, in this case we were able to
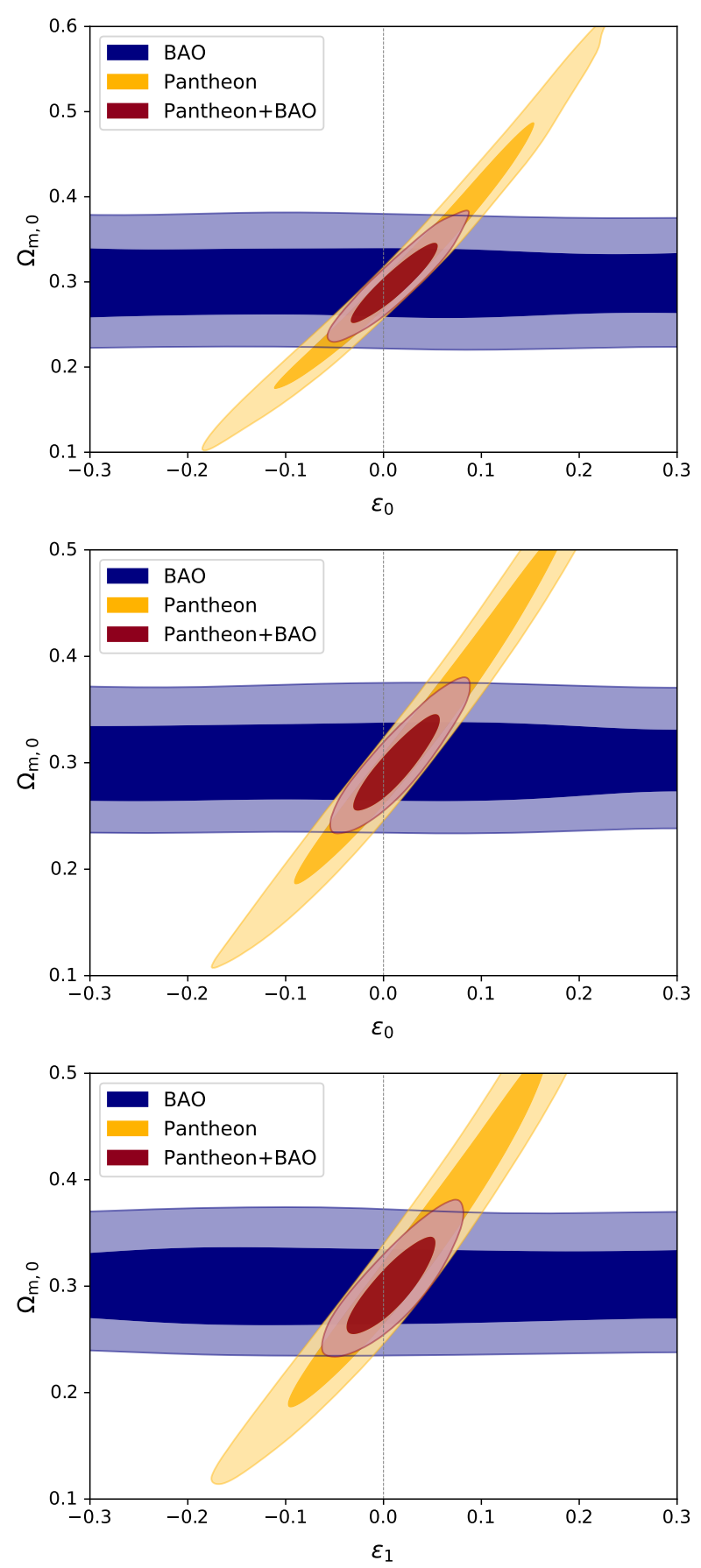

Fig. 1. 2D contours on $\Omega_{\mathrm{m}, 0}, \epsilon_{0}$ and $\epsilon_{1}$, using currently available data for BAO (blue), SnIa (yellow) and the combination of the two (red). These results refer to the constant (top panel) and binned (central and bottom panels) $\epsilon(z)$ cases.

find a compact expression for the GA reconstruction of the $\eta(z)$ parameter, given by

$\eta(z)=(1+z)^{0.0294-0.0002 z^{4}}$.

As can be seen, the value of $\epsilon(z)$ derived from Eq. (18) is compatible with the one derived in the parameterized approach. Moreover, the value predicted from the GAs has a redshift dependence $O\left(z^{4}\right)$, which is only important at high redshifts as the coefficient is sufficiently small, albeit negative. This is also in agreement with the fact that the parameterized approach finds a value for $\epsilon$ 


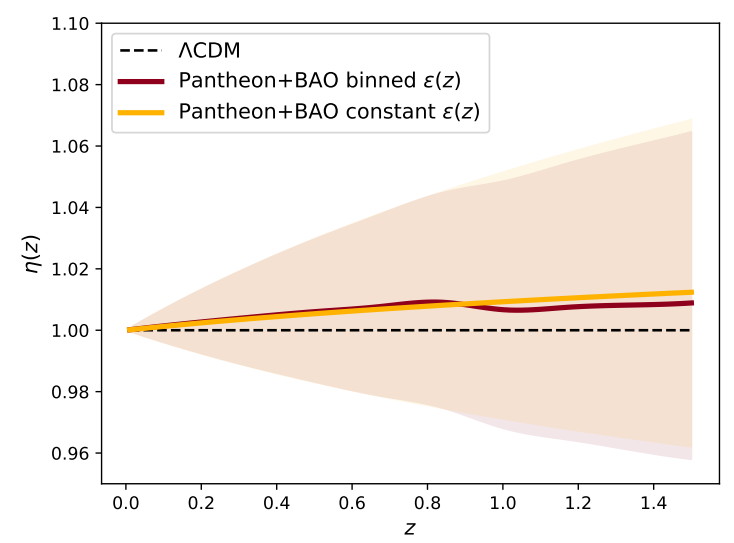

Fig. 2. Reconstruction with current SnIa and BAO data of the $\eta(z)$ function at different redshifts as derived parameters using Eq. (4). The mean function is shown as a solid line, while the shaded area represents the $68 \%$ confidence region. The red colour shows the result in the binned $\epsilon(z)$ case, while yellow refers to the constant case.

that is smaller in the second bin with respect to the first. Finally, the GAs reconstructions for the luminosity distance or the Hubble parameter are unfortunately far too unwieldy, so we refrain from reporting them here.

Having performed the fit to the data, we now show in the left panel of Fig. 3 the reconstruction of the distance modulus $\mu(z)=m(z)-M_{0}$, rescaled by the $\Lambda$ CDM best-fit with $\Omega_{\mathrm{m}, 0}=$ $0.297 \pm 0.018$. By definition, the dashed line at zero corresponds to the best-fit $\Lambda \mathrm{CDM}$, the red line is the GAs fit and the shaded region corresponds to the $1 \sigma \mathrm{GA}$ errors. The constraints on the distance modulus are tighter at low redshifts $z \in[0,0.5]$ where we have the bulk of the BAO and SnIa data points, but also due to the fact that the distance modulus $\mu(z)$ is a function of the luminosity distance $d_{\mathrm{L}}(z)$ and as a result it naturally converges to a fixed value at $z=0$.

In the right panel of Fig. 3 we show the duality parameter $\eta(z)$ of Eq. (4), obtained through the GAs reconstruction of $d_{\mathrm{A}}(z)$ from the BAO data and the luminosity distance $d_{\mathrm{L}}(z)$ based on the Pantheon SnIa set. The dashed line at unity is $\Lambda \mathrm{CDM}$, the red line is the GAs fit and the shaded region corresponds to the $1 \sigma$ GAs errors. As can be seen, the reconstruction of $\eta(z)$ is compatible with unity at the $1 \sigma$ level, hence the GAs do not detect any statistically significant deviations from the $\Lambda$ CDM model with the currently available data.

\section{Snla and BAO mock data}

Upcoming surveys have the potential to improve the constraining power on deviations from the standard DDR, thanks to improved data both for SnIa and LSS observations, where the latter provide information on the angular diameter distance and the Hubble parameter. We are interested therefore in forecasting how future surveys will constrain the DDR and in order to do so we create simulated datasets for both SnIa and BAO measurements.

For these simulations, we use the fiducial cosmology shown in Table 2, that is the same used in EC19, where we assume no violation of the DDR. Using these values we create our fiducial luminosity and angular diameter distances, as well as the redshift evolution of the Hubble parameter. Once the fiducial cosmological quantities are computed, we create our mock data following the specification of forthcoming surveys.

It is important to mention that given the high precision expected from future surveys, it will be even more important to ensure their accuracy. Several analyses are being performed to understand the observational systematic uncertainties that will affect future measurements. In Euclid Collaboration (2020b), for instance, a detailed analysis on the observational systematic effects related to the Euclid VIS instrument is performed, including charge transfer ineffiency and modelling of the point spread function. Given that the specifications for the future instruments and the modelling of their systematic effects might still evolve during their completion, in this work we assume that the observational systematic effects will be under control when the data arrive. Nevertheless, we do include astrophysical systematic effects, like galaxy bias, as described in the following subsections.

\subsection{Snla surveys}

Here we consider two different surveys. On the one hand, we simulate future observations based on the specifications of the LSST, which we assume will observe a number of SnIa $N_{\text {SnIa }}=$ 8800 in the redshift range $z \in[0.1,1.0]$. We then extend the redshift range of our SnIa dataset by including simulated observations for the proposed Euclid DESIRE survey (Laureijs et al. 2011; Astier et al. 2014), thus including 1700 additional data points in the range $z \in[0.7,1.6]$. For both surveys, we assume the redshift distributions shown in Astier et al. (2014) and we further assume that the two are not correlated ${ }^{2}$. For each event, we simulate an observational error $\sigma_{\text {tot }, i}$ given by

$\sigma_{\text {tot }, i}^{2}=\delta \mu_{i}^{2}+\sigma_{\text {flux }}^{2}+\sigma_{\text {scat }}^{2}+\sigma_{\text {intr }}^{2}$,

where the flux, scatter, and intrinsic contributions are the same for each event $\left(\sigma_{\text {flux }}=0.01, \sigma_{\text {scat }}=0.025\right.$, and $\sigma_{\text {intr }}=0.12$, respectively) and we add an error contribution on the distance modulus $\mu=m-M$, which evolves linearly in redshift

$\delta \mu=e_{M} z$

where $e_{M}$ is drawn from a Gaussian distribution with vanishing mean and $\sigma\left(e_{M}\right)=0.01$ (see Gong et al. 2010; Astier et al. 2014).

We note that while the effects of lensing by foreground structures are already included in the Pantheon data by incorporating an error $\sigma_{\text {lens }}$ (see Scolnic et al. 2018), here we have not included this error in our mocks as it has a very weak redshift dependence and is subdominant with respect to the intrinsic distance scatter of every point of $\sim 0.12$ mag. Thus, we do not expect it to affect our results.

\subsection{LSS surveys}

One of the main objectives of this paper is to forecast the constraints achievable on DDR with Euclid. As such, we simulate BAO data from this survey using the Fisher matrix technique, following the same strategy used in EC19 for the spectroscopic survey.

Since in this work we are interested in using precise measurements of the Hubble parameter and the angular diameter distance to test the DDR, we will focus on the spectroscopic Euclid survey. Through this, Euclid will be capable of exploring the galaxy power spectrum in a range of redshifts $z \in[0.95,1.75]$.

2 We stress that the DESIRE survey is not a guaranteed output of Euclid. Here we include this in the analysis as a possible survey extending the redshift range of LSST. Such a survey will be crucial for performing the GA reconstruction at higher redshifts. 

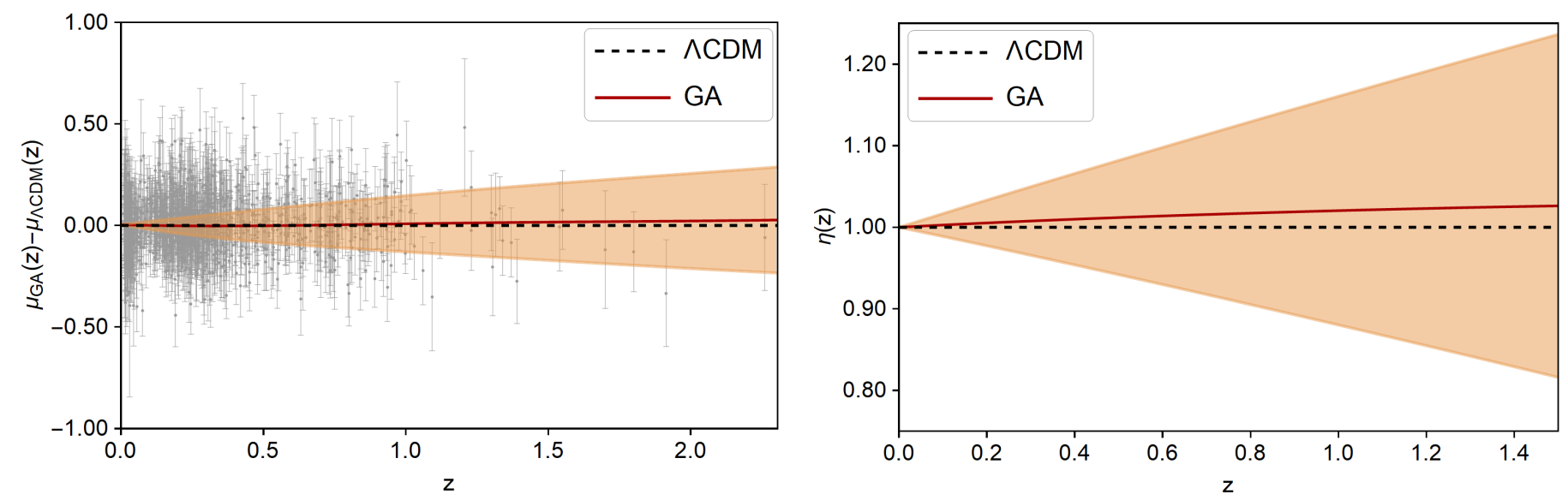

Fig. 3. Left: distance modulus based on the Pantheon SnIa set, rescaled by the best-fit $\Lambda$ CDM model $\left(\Omega_{\mathrm{m}, 0}=0.297 \pm 0.018\right)$. The dashed line at zero corresponds to $\Lambda \mathrm{CDM}$, the red line is the GA fit, and the shaded region corresponds to the $1 \sigma$ GA errors. Right: duality relation $\eta(z)$ for the GA reconstruction of $d_{\mathrm{A}}(z)$ from the BAO data and the luminosity distance $d_{\mathrm{L}}(z)$ based on the Pantheon SnIa set. The dashed line at unity is $\Lambda \mathrm{CDM}$, the red line is the GA fit, and the shaded region is the $1 \sigma \mathrm{GA}$ errors.

Table 2. Parameter values for the fiducial model we used for the mock.

\begin{tabular}{lccccccc}
\hline \hline$M_{0}$ & $\Omega_{\mathrm{m}, 0}$ & $\Omega_{\mathrm{b}, 0} h^{2}$ & $H_{0}$ & $w_{0}$ & $w_{a}$ & $\epsilon_{0}$ & $\epsilon_{1}$ \\
\hline-19.3 & 0.32 & 0.02225 & 67 & -1 & 0 & 0 & 0 \\
\hline
\end{tabular}

Notes. The values used follow the fiducial of EC19. $H_{0}$ is shown in units of $\mathrm{km} \mathrm{s}^{-1} \mathrm{Mpc}^{-1}$.

As described in EC19, the main targets are $\mathrm{H}_{\alpha}$ emitters and the survey is able to measure up to 30 million spectroscopic redshifts with an error of $\sigma_{z}=0.001(1+z)$ (Pozzetti et al. 2016). The main observable is the galaxy power spectrum which contains information about the galaxy bias, the anisotropies due to redshift space distortions, the residual shot noise, the redshift uncertainty and the distortion due to the Alcock-Paczynski effect. Furthermore, the matter power spectrum has been modulated with non-linear effects which distort the shape of the power spectrum (Wang et al. 2013).

With respect to EC19, in this work we use a different binning scheme. Instead of four redshift bins we divide the observed redshift range in nine equally spaced bins of width $\Delta z=0.1$. The galaxy number density $n(z)$, in units of $\mathrm{Mpc}^{-3}$ and the galaxy bias $b(z)$ have been obtained rebinning those of EC19, finding:

$$
\begin{aligned}
& n(z)=\{2.04,2.08,1.78,1.58,1.39,1.15,0.97,0.7,0.6\} \times 10^{-4} \\
& b(z)=\{1.42,1.5,1.57,1.64,1.71,1.78,1.84,1.90,1.96\} .
\end{aligned}
$$

The different binning choice allows obtaining more data points from this survey, which improves the machine learning analysis we perform through GA. Nevertheless, we have compared the final bounds obtained on cosmological parameters with this choice against those of EC19, finding no significant effect.

Using these specifications, we follow the procedure described in EC19 to obtain the Fisher matrix for the full set of cosmological parameters, namely: four shape parameters $\left\{\omega_{\mathrm{m}}=\Omega_{\mathrm{m}, 0}\right.$ $\left.h^{2}, h, \omega_{\mathrm{b}}=\Omega_{\mathrm{b}, 0} h^{2}, n_{s}\right\}$, two non-linear parameters $\left\{\sigma_{\mathrm{p}}, \sigma_{\mathrm{v}}\right\}$ and five redshift dependent parameters $\left\{\ln d_{\mathrm{A}}, \ln H, \ln f \sigma_{8}\right.$, $\left.\ln b \sigma_{8}, P_{\mathrm{s}}\right\}$ evaluated in each redshift bin. Using such an approach, we obtain the expected errors from this survey on the angular diameter distance $d_{\mathrm{A}}(z)$ and the Hubble parameter $H(z)$ in each of the nine redshift bins, while marginalizing over all the other free parameters. The results of the Fisher matrix procedure are in principle dependent on the chosen fiducial cosmology, but we assume here that this dependence is negligible.

In Sect. 4 we have shown how for our parameterized approach we need to break the degeneracy between the DDR parameters and $\Omega_{\mathrm{m}, 0}$, and the BAO measurements from Euclid will be able to measure this parameter. However, we are able to use our GA reconstruction approach only in the redshift range where both SnIa and BAO data are available. Using only Euclid alongside LSST and DESIRE would therefore limit the validity of such an approach to only the redshift range $z \in[0.95,1.6]$. In order to be able to reconstruct the DDR functions at all redshift for which we have SnIa data available, we complement the redshift range of Euclid by exploiting the extended redshift range of the DESI survey, which started operations at the end of 2019 and will obtain optical spectra for tens of millions of galaxies and quasars up to redshift $z \sim 4$.

Such spectra will enable BAO and redshift-space distortion cosmological analyses. We use here the official DESI forecasts on future constraints for both $H(z)$ and $d_{\mathrm{A}}(z)$ (DESI Collaboration 2016). These have been obtained with a Fisher matrix formalism, following Font-Ribera et al. (2014), which includes the "broadband" galaxy power, meaning measurements of the power spectrum as a function of redshift, wavenumber, and angle with respect to the line of sight. As for the Euclid approach described above, this encodes all the available information from the two-point clustering and not just the position of the BAO peak. In more detail, we consider the DESI baseline survey, which consists of a coverage of $14000 \mathrm{deg}^{2}$ and the four different types of DESI targets: bright galaxies (BGs), luminous red galaxies (LRGs), emission line galaxies (ELGs), and quasars. The DESI forecast measurements will cover the redshift range $z \in[0.05,3.55]$, but their precision will also depend on the target population. The BGs will cover the redshift range $z \in[0.05,0.45]$ in five equispaced redshift bins, the LRGs and ELGs will focus on $z \in[0.65,1.85]$ with 13 equispaced redshift bins, while the Ly- $\alpha$ forest quasar survey will cover $z \in[1.96,3.55]$ with 11 equispaced redshift bins. We further assume these measurements to be uncorrelated.

In the following, when using the combination of BAO data from Euclid and DESI, as we do not consider correlations between these surveys, we will only include DESI observations that do not overlap in redshift with the Euclid measurements. 
Table 3. Mean values and marginalized $68 \%$ confidence level errors obtained from mock LSST (SnIa), Euclid (SnIa and BAO) and DESI (BAO) data on the cosmological parameters $\Omega_{\mathrm{m}, 0}$ and $H_{0}$ (in units of $\mathrm{km} \mathrm{s}^{-1} \mathrm{Mpc}^{-1}$ ) and on the DDR parameters $\epsilon_{0}$ and $\epsilon_{1}$ (if present).

\begin{tabular}{lccc}
\hline \hline & & Const. $\epsilon(z)$ & Binned $\epsilon(z)$ \\
\hline Param. & Probe & & \\
\hline \multirow{4}{*}{$H_{0}$} & BAO & $67.13 \pm 0.25$ & $67.14 \pm 0.25$ \\
& SnIa & Unconstrained & Unconstrained \\
& SnIa+BAO & $67.14 \pm 0.26$ & $67.15 \pm 0.26$ \\
\hline \multirow{4}{*}{$\Omega_{\mathrm{m}, 0}$} & BAO & $0.3175 \pm 0.0034$ & $0.3174 \pm 0.0034$ \\
& SnIa & $0.259 \pm 0.077$ & $0.281 \pm 0.096$ \\
& SnIa+BAO & $0.3174_{-0.0036}^{+0.0032}$ & $0.3172 \pm 0.0035$ \\
\hline \multirow{4}{*}{$\epsilon_{0}$} & BAO & Unconstrained & Unconstrained \\
& SnIa & $-0.060_{-0.062}^{+0.090}$ & $-0.040_{-0.071}^{+0.11}$ \\
& SnIa+BAO & $-0.0008 \pm 0.0049$ & $0.0019 \pm 0.0061$ \\
\hline \multirow{4}{*}{$\epsilon_{1}$} & BAO & - & Unconstrained \\
& SnIa & - & $-0.042_{-0.068}^{+0.11}$ \\
& SnIa+BAO & - & $0.0001 \pm 0.0049$ \\
\hline
\end{tabular}

Moreover, since we only have SnIa data from LSST+DESIRE up to $z=1.6$, we will only include in the analysis the full BGs survey and the LRGs and ELGs up to $z=0.9$, thus including no information from observations of the Ly- $\alpha$ forest.

\section{Forecast results}

Following the approach described in Sect. 3, we constrain the cosmological and DDR parameters using our mock data for SnIa and BAO. In Table 3 we show the mean values and errors for the free parameters of the analysis, when using the data from LSST+DESIRE for SnIa and from Euclid+DESI for BAO, for both the constant and binned $\epsilon(z)$. In Fig. 4 instead we compare the results of the combination of these surveys with those obtained using current data and to what can be achieved using only the BAO survey from Euclid. This is to be compared with Fig. 54 in Amendola et al. (2018), whose forecast is for Euclid (with the specifications foreseen at the time) plus a Stage IV (SNAP-like) SnIa mission: the achieved constraints are compatible with what we find here. In summary, $\epsilon_{0}$ and $\epsilon_{1}$ are now constrained with an error smaller than $10^{-2}$, improving the sensitivity of current constraints by about a factor of $\approx 6$. We notice that here we are not considering one of the two primary probes of Euclid, that is cosmic shear. Adding the information brought by such a probe would further constrain the value of $\Omega_{\mathrm{m}, 0}$, thus resulting in even tighter bounds on the DDR parameters $\epsilon_{0}$ and $\epsilon_{1}$.

As can be seen in Fig. 4 the addition of DESI to the combination of LSST+Euclid does not improve the constraints significantly. This could be somewhat surprising, as one would expect that, given the complementarity in redshift range between the two surveys, the combination of the two would provide improvements in the constraints. Our results show instead that the constraining power on $\Omega_{\mathrm{m}, 0}$ from Euclid alone is the one dominating the constraints and therefore driving the breaking of the degeneracy between $\Omega_{\mathrm{m}, 0}$ and $\epsilon(z)$ parameters. While the $z=0.9$ cut we perform to avoid an overlap of the two BAO surveys is not per se behind this effect, we do expect that the use of the full DESI data (specifically the high-redshift galaxy and the Ly- $\alpha$ data) would lead to a stronger improvement in the constraints. However, in order to investigate this further, cor-
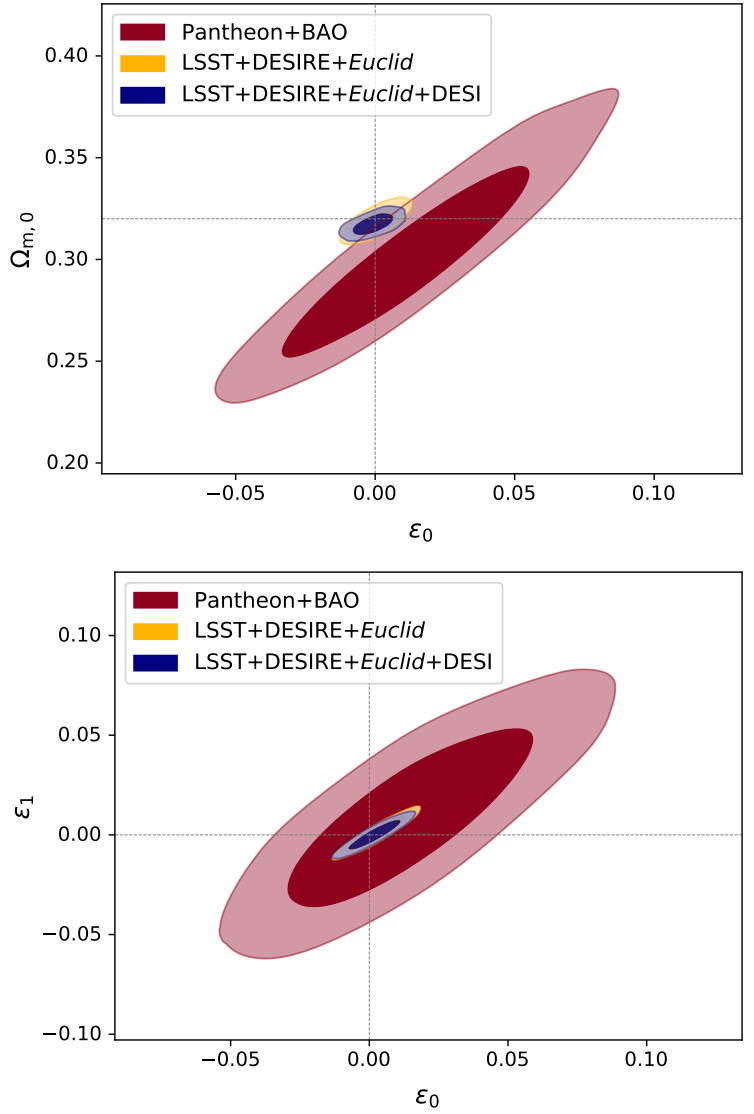

Fig. 4. $2 \mathrm{D}$ contours on $\Omega_{\mathrm{m}, 0}, \epsilon_{0}$ and $\epsilon_{1}$, using the combination of BAO and SnIa dataset given by currently available data (red contours), LSST supernovae and Euclid BAO data (yellow contours), and the combination of LSST supernovae with BAO forecasts coming from the combination of Euclid and DESI (blue contours). These results refer to the constant (top panel) and binned (bottom panel) $\epsilon(z)$ cases. The dashed lines identify the limit $\epsilon(z)=0$.

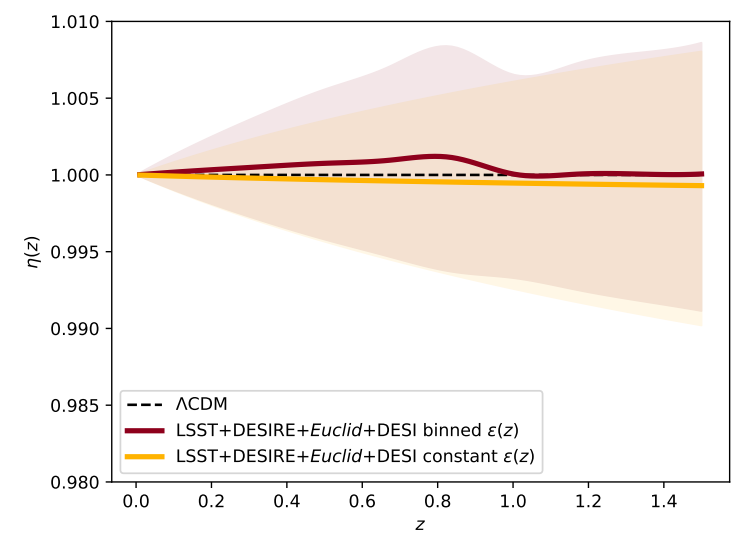

Fig. 5. Reconstruction with future SnIa and BAO data of the $\eta(z)$ function at different redshifts, as derived parameters using Eq. (4). The mean function is shown as a solid line, while the shaded area represents the $68 \%$ confidence region. The red colour shows the result in the binned $\epsilon(z)$ case, while yellow refers to the constant case.

relations between the two mock datasets should be properly taken into account, an analysis that is outside the scope of this paper.

Furthermore, in Fig. 5 we show $\eta(z)$ obtained using Eq. (4) with the parameterized approach constraints; we find that these 

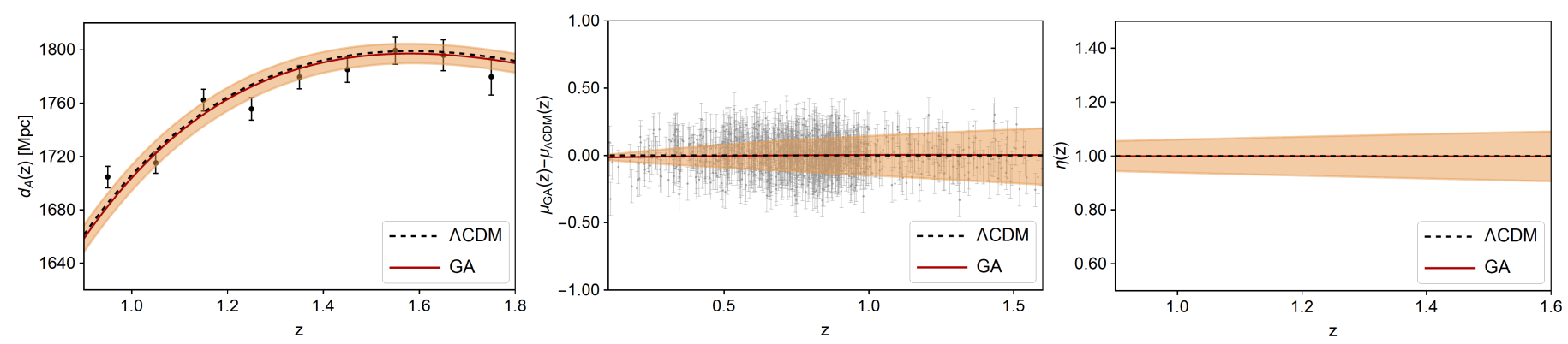

Fig. 6. Left: reconstruction of the angular diameter distance $d_{\mathrm{A}}(z)$ from the mock Euclid data with the GA approach. The dashed black line is $\Lambda \mathrm{CDM}$, the solid red line is the GA fit and the shaded region corresponds to the $1 \sigma$ GA errors. Centre: results on the distance modulus $\mu(z)$ from the mock Euclid+LSST+DESIRE data with the GA approach. The dashed line at zero is $\Lambda$ CDM, the red line is the GA fit and the shaded region corresponds to the $1 \sigma$ GA errors. For clarity, we only show one thousand of the total SnIa points. Right: reconstruction of the $\eta(z)$ parameter in the range $z \in[0.9,1.6]$. In all cases the error bars of the data points correspond to $1 \sigma$ uncertainty.
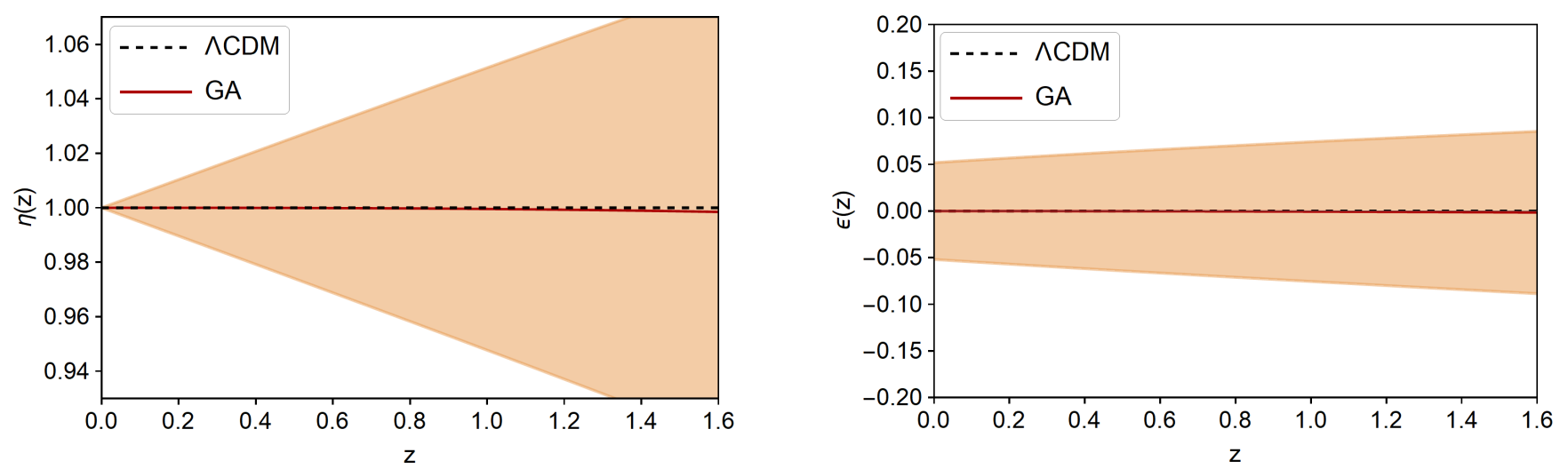

Fig. 7. Left: duality relation $\eta(z)$ for the GA reconstructions for the LSST+DESIRE SnIa data and Euclid plus DESI BAO mocks. Right: reconstruction of the $\epsilon(z)$ parameter, calculated via $\epsilon(z)=\frac{\ln \eta(z)}{\ln (1+z)}$, is found to be consistent with the fiducial value $\epsilon(z)=0$ within the errors. In both cases the GA reconstruction is the red line, while the shaded region corresponds to the $1 \sigma$ errors.

are significantly improved with respect to current results, showing the high constraining power that can be reached using upcoming surveys.

As a final analysis, we apply the GAs reconstruction method to the mock BAO and SnIa data. In the left panel of Fig. 6 we show the reconstruction of the angular diameter distance $d_{\mathrm{A}}(z)$ with the GA approach using the mock BAO Euclid data. The dashed black line is the best-fit $\Lambda$ CDM model, the solid red line is the GAs fit to the Euclid BAO data, while the shaded region corresponds to the $1 \sigma$ GA errors. In the central panel of Fig. 6 we show the GAs reconstruction of the distance modulus $\mu(z)$ using the LSST+DESIRE simulated data rescaled by the best-fit $\Lambda C D M$, with the latter corresponding to the dashed line at zero. Similarly to the left panel, the red line is the GAs fit and the shaded region corresponds to the $1 \sigma$ GAs errors. For clarity, we only show 1000 out of the total $\sim 10000$ SnIa points we include in our mock dataset.

Finally, in the right panel of Fig. 6 we show the reconstruction of the $\eta(z)$ function, which is obtained from the joint GA reconstruction of $d_{\mathrm{A}}(z)$ and $d_{\mathrm{L}}(z)$. It is immediately clear that such a reconstruction is limited if performed using Euclid BAO data only: the lack of $d_{\mathrm{A}}(z)$ measurements for $z<0.9$ forces the method to be applied only in the redshift range $z \in[0.9,1.6]$. Despite this, as can be seen in all panels of Fig. 6, the GA reconstruction for the mock Euclid BAO recovers the correct fiducial model in all three cases and in particular, it provides very tight constraints on both the angular diameter distance and the duality parameter $\eta(z)$. Specifically, comparing with the right panel of
Fig. 3, we can see that with the GA approach, Euclid now brings roughly a factor of three improvement compared to the current data with a non-parametric approach.

We also consider the added benefit on the reconstruction brought by DESI BAO data, which cover, as mentioned earlier, the redshift range $z \in[0,0.9]$. In Fig. 7 we show the reconstruction of the duality relation $\eta(z)$ (left) and the $\epsilon(z)$ parameter (right) when the full data combination of Euclid and DESI is considered. We find that, as in the case of the parameterized results, the addition of DESI to the combination of LSST +Euclid does not improve the constraints significantly, however in this case we can now cover a wider redshift range. The GA can then recover the fiducial model $\eta(z)=1$ with a $1 \%$ error at $z=0.2$ and a $5 \%$ error at $z=1$, while the parameter $\epsilon(z)$, obtained inverting Eq. (4), can be measured in a model-independent fashion, with an error between 0.05 and 0.07 over the redshift range covered by the data.

\section{Conclusions}

In this work, we have constrained deviations from the standard DDR using current and forecast data; for the latter, we focused mainly on the constraints achievable through synergies between Euclid and contemporary surveys, both for SnIa and BAO. We have discussed in Sect. 2 several physical mechanisms that can lead to a violation of the DDR, both of astrophysical and BSM origin. For this reason, while we exploited a commonly used parametric approach to constrain the DDR breaking function 
$\epsilon(z)$, we also used a fully agnostic reconstruction through GAs The latter approach allows us to obtain constraints without any assumption on the redshift trend of possible deviations from the standard theory.

In the parametric case, within the assumption of a flat $\Lambda$ CDM expansion and using the value of $\Omega_{\mathrm{b}, 0} h^{2}$ measured by Planck, we found that current SnIa data loosely constrain the deviation from the $\epsilon(z)=0$ limit, due to the degeneracy between the DDR parameters and the total matter energy density $\Omega_{\mathrm{m}, 0}$. Such a degeneracy is broken when including BAO data; these are not sensitive to violations of DDR, but tightly constrain $\Omega_{\mathrm{m}, 0}$, thus yielding tight constraints on the parameterized $\epsilon(z)$. The results obtained with the combination of SnIa and BAO data show that the standard DDR is within $\approx 1 \sigma$ and the results are compatible with a constant $\epsilon(z)$.

In the case of the machine learning reconstruction, we found that the GA can provide robust constraints in line with the parametric approach, albeit with somewhat larger uncertainties. This is due to the fact that the GA is non-parametric, thus it provides broader and theory-agnostic constraints. Specifically, we found that in the case of the currently available data, the reconstruction of the duality parameter $\eta(z)$ was fully consistent with the parametric approach and with unity.

Using the same fiducial cosmology assumed in EC19, we have then created simulated data for upcoming surveys; we focused mainly on the BAO data achievable with Euclid and on the possible SnIa survey DESIRE that might be provided by this satellite. We complemented the redshift range of Euclid forecast data with contemporary surveys, namely LSST for SnIa and DESI for BAO.

Analysing these mock data through the parameterized approach, we found an improvement of a factor $\approx 6$ with respect to current results when the combination of SnIa and BAO is considered (see Table 3). We have also shown in Fig. 5 how such constraints translate into a redshift trend for the $\eta(z)$ function, highlighting how the use of mock data significantly improves the bounds on this function, which is now constrained to vary by less than $1 \%$ from the fiducial assumption of $\eta(z)=1$.

Using the GA with the mock data, we reconstructed the duality parameter $\eta(z)$ and we have shown how synergies of Euclid with other galaxy surveys are crucial in order to be able to use such an approach over an extended range in redshift. With the data combinations considered here we found that the GA can recover the fiducial model $\eta(z)=1$ with an error of $1 \%$ at $z=0.2$ and of $5 \%$ at $z=1$, as shown in Fig. 7, and with an improvement of roughly a factor of three over the current DDR constraints in the same redshift range. This somewhat less constraining result, compared to the parameterized approach, is mainly due to the completely model-independent and theoryagnostic approach employed here, despite the joint fitting of the SnIa and BAO data.

In summary, our paper highlighted the benefits of synergies between the Euclid BAO survey and external probes in constraining physics beyond the standard model, which could manifest itself through violations of the DDR. In particular, we have demonstrated that such a BAO survey will make it possible to constrain deviations from the DDR at an unprecedented level in the near future using parameterized approaches, while it will also reach a high enough sensitivity to employ modelindependent approaches that allow an agnostic reconstruction of possible deviations from the standard DDR.
Acknowledgements. We are grateful to P. Astier for useful discussions and to T. Baker, G. Castignani and P. Ntelis for comments on the manuscript. MM has received the support of a fellowship from "la Caixa" Foundation (ID 100010434), with fellowship code LCF/BQ/PI19/11690015, and the support of the Spanish Agencia Estatal de Investigacion through the grant "IFT Centro de Excelencia Severo Ochoa SEV-2016-0597". The work of CJM was financed by FEDER - Fundo Europeu de Desenvolvimento Regional funds through the COMPETE 2020 - Operational Programme for Competitiveness and Internationalisation (POCI), and by Portuguese funds through FCT - Fundação para a Ciência e a Tecnologia in the framework of the project POCI-01-0145-FEDER028987. S.N. acknowledges support from the research project PGC2018094773-B-C32, the Centro de Excelencia Severo Ochoa Program SEV-2016059 and the Ramón y Cajal program through Grant No. RYC-2014-15843. D.S. acknowledges financial support from the Fondecyt Regular project number 1200171. I.T. acknowledges support from the Spanish Ministry of Science, Innovation and Universities through grant ESP2017-89838-C3-1-R, and the H2020 programme of the European Commission through grant 776247. A.A. acknowledges support from the Science and Technology Facilities Council (STFC) grant ST/P000703/1. V.Y. acknowledges funding from the European Research Council (ERC) under the European Union's Horizon 2020 research and innovation programme (grant agreement No. 769130) The Euclid Consortium acknowledges the European Space Agency and a number of agencies and institutes that have supported the development of Euclid, in particular the Academy of Finland, the Agenzia Spaziale Italiana, the Belgian Science Policy, the Canadian Euclid Consortium, the Centre National d'Etudes Spatiales, the Deutsches Zentrum für Luft- und Raumfahrt, the Danish Space Research Institute, the Fundação para a Ciência e a Tecnologia, the Ministerio de Economia y Competitividad, the National Aeronautics and Space Administration, the Netherlandse Onderzoekschool Voor Astronomie, the Norwegian Space Agency, the Romanian Space Agency, the State Secretariat for Education, Research and Innovation (SERI) at the Swiss Space Office (SSO), and the United Kingdom Space Agency. A complete and detailed list is available on the Euclid web site (http://www . euclid-ec.org).

\section{References}

Abbott, T. M. C., Abdalla, F. B., Alarcon, A., et al. 2019, MNRAS, 483, 4866 Aguirre, A. N. 1999, ApJ, 525, 583

Aguirre, A., \& Haiman, Z. 2000, ApJ, 532, 28

Ahlers, M. 2009, PRD, 80, 023513

Akrami, Y., Scott, P., Edsjo, J., Conrad, J., \& Bergstrom, L. 2010, JHEP, 04, 057

Albrecht, A., Bernstein, G., Cahn, R., et al. 2006, ArXiv e-prints [arXiv:astro-ph/0609591]

Amendola, L., Appleby, S., Avgoustidis, A., et al. 2018, Liv. Rev. Relat., 21, 2 Anderson, L., Aubourg, E., Bailey, S., et al. 2014, MNRAS, 441, 24

Angulo, R., Baugh, C., Frenk, C., \& Lacey, C. 2008, MNRAS, 383, 755

Anselmi, S., Starkman, G. D., Corasaniti, P.-S., Sheth, R. K., \& Zehavi, I. 2018, Phys. Rev. Lett., 121, 021302

Arjona, R. 2020, JCAP, 08, 009

Arjona, R., \& Nesseris, S. 2020a, Phys. Rev. D, 101, 123525

Arjona, R., \& Nesseris, S. 2020b, J. Cosmol. Astropart. Phys., 11, 042

Astier, P., Balland, C., Brescia, M., et al. 2014, A\&A, 572, A80

Ata, M., Baumgarten, F., Bautista, J., et al. 2018, MNRAS, 473, 4773

Avgoustidis, A., Verde, L., \& Jimenez, R. 2009, JCAP, 06, 012

Avgoustidis, A., Burrage, C., Redondo, J., Verde, L., \& Jimenez, R. 2010, JCAP, 10,024

Avgoustidis, A., Luzzi, G., Martins, C. J. A. P., \& Monteiro, A. M. R. V. L. 2012, JCAP, 02, 013

Avgoustidis, A., Martins, C. J. A. P., Monteiro, A. M. R. V. L., Vielzeuf, P. E., \& Luzzi, G. 2014, JCAP, 06, 062

Avgoustidis, A., Génova-Santos, R. T., Luzzi, G., \& Martins, C. J. A. P. 2016, PRD, 93, 043521

Bassett, B. A., \& Kunz, M. 2004, PRD, 69, 101305

Batell, B., \& Gherghetta, T. 2006, PRD, 73, 045016

Bautista, J. E., Vargas-Magaña, M., Dawson, K. S., et al. 2018, ApJ, 863, 110

Beutler, F., Blake, C., Colless, M., et al. 2011, MNRAS, 416, 3017

Blake, C., Brough, S., Colless, M., et al. 2012, MNRAS, 425, 405

Blomqvist, M., du Mas des Bourboux, H., \& Busca, N. G. 2019, A\&A, 629, A86

Bogdanos, C., \& Nesseris, S. 2009, JCAP, 05, 006

Brax, P., Burrage, C., Davis, A.-C., Seery, D., \& Weltman, A. 2010, PRD, 81, 103524

Burrage, C. 2008, PRD, 77, 043009

Conley, A., Guy, J., Sullivan, M., et al. 2011, ApJS, 192, 1

Corasaniti, P. S. 2006, MNRAS, 372, 191

Costille, A., Caillat, A., Rossin, C., et al. 2018, in Space Telescopes and Instrumentation 2018: Optical, Infrared, and Millimeter Wave, eds. M. 
Lystrup, H. A. MacEwen, G. G. Fazio, et al., Int. Soc. Opt. Photonics (SPIE), 10698, 730

Cropper, M., Pottinger, S., Azzollini, R., et al. 2018, in Space Telescopes and Instrumentation 2018: Optical, Infrared, and Millimeter Wave, eds. M. Lystrup, H. A. MacEwen, G. G. Fazio, et al., Int. Soc. Opt. Photonics (SPIE), 10698, 709

Csáki, C., Kaloper, N., \& Terning, J. 2002, PRL, 88, 161302

Cuesta, A. J., Verde, L., Riess, A., \& Jimenez, R. 2015, MNRAS, 448, 3463

DESI Collaboration (Aghamousa, A., et al.) 2016, ArXiv e-prints [arXiv:1611.00036]

Eisenstein, D. J., \& Hu, W. 1998, ApJ, 496, 605

Etherington, I. M. H. 1933, Philos. Mag., 15, 761

Euclid Collaboration (Blanchard, A., et al.) 2020a, A\&A, 642, A191

Euclid Collaboration (Paykari, P., et al.) 2020b, A\&A, 635, A139

Font-Ribera, A., McDonald, P., Mostek, N., et al. 2014, JCAP, 05, 023

Gil-Marín, H., Percival, W. J., Cuesta, A. J., et al. 2016, MNRAS, 460, 4210

Gong, Y., Cooray, A., \& Chen, X. 2010, ApJ, 709, 1420

Hogg, N. B., Martinelli, M., \& Nesseris, S. 2020, ArXiv eprints [arXiv:2007.14335]

Holanda, R., Lima, J., \& Ribeiro, M. 2010, ApJ, 722, L233

Holanda, R., Gonçalves, R., \& Alcaniz, J. 2012, JCAP, 06, 022

Holdom, B. 1986, Phys. Lett. B, 166, 196

Jaeckel, J., \& Ringwald, A. 2010, Ann. Rev. Nucl. Part. Sci., 60, 405

Kowalski, M., Rubin, D., Aldering, G., et al. 2008, ApJ, 686, 749

Laureijs, R., Amiaux, J., Arduini, S., et al. 2011, ArXiv e-prints [arXiv:1110.3193]

Leite, A. C. O., Martins, C. J. A. P., Molaro, P., Corre, D., \& Cristiani, S. 2016, PRD, 94, 123512

Li, Z., Wu, P., \& Yu, H. W. 2011, ApJ, 729, L14

Liao, K. 2019, ApJ, 885, 70

Liao, K., Li, Z., Cao, S., et al. 2016, ApJ, 822, 74

Lima, J. A. S., Silva, A. I., \& Viegas, S. M. 2000, MNRAS, 312, 747

Liske, J., et al. 2014, Top Level Requirements For ELT-HIRES, Document ESO 204697 Version 1

LSST Science Collaboration (Abell, P. A., et al.) 2009, ArXiv e-prints [arXiv:0912.0201]

Luzzi, G., Shimon, M., Lamagna, L., et al. 2009, ApJ, 705, 1122

Lv, M.-Z., \& Xia, J.-Q. 2016, Phys. Dark Univ., 13, 139

Ma, C., \& Corasaniti, P.-S. 2018, ApJ, 861, 124

Maartens, R. 2011, Philos. Trans. R. Soc. Lond. A, 369, 5115

Ménard, B., Nestor, D., Turnshek, D., et al. 2008, MNRAS, 385, 1053

More, S., Bovy, J., \& Hogg, D. W. 2009, ApJ, 696, 1727

Mörtsell, E., Bergström, L., \& Goobar, A. 2002, PRD, 66, 047702

Nesseris, S., \& Garcia-Bellido, J. 2012, JCAP, 11, 033

Nesseris, S., \& Garcia-Bellido, J. 2013, PRD, 88, 063521

Nesseris, S., \& Shafieloo, A. 2010, MNRAS, 408, 1879

Noterdaeme, P., Petitjean, P., Srianand, R., Ledoux, C., \& López, S. 2011, A\&A 526, L7

Ntelis, P., Hamilton, J.-C., Le Goff, J.-M., et al. 2017, JCAP, 06, 019

Pepe, F., Cristiani, S., Rebolo, R., et al. 2013, The Messenger, 153, 6

Planck Collaboration VI. 2020, A\&A, 641, A6

Pozzetti, L., Hirata, C. M., Geach, J. E., et al. 2016, A\&A, 590, A3

Ross, A. J., Samushia, L., Howlett, C., et al. 2015, MNRAS, 449, 835

Sapone, D., Majerotto, E., \& Nesseris, S. 2014, PRD, 90, 023012

Scolnic, D. M., Jones, D. O., Rest, A., et al. 2018, ApJ, 859, 101

Stern, D., Jimenez, R., Verde, L., Kamionkowski, M., \& Stanford, S. 2010, JCAP, 02,008

Svrcek, P., \& Witten, E. 2006, JHEP, 06, 051

Torrado, J., \& Lewis, A. 2020, ArXiv e-prints [arXiv:2005.05290]

Wang, Y., Chuang, C.-H., \& Hirata, C. M. 2013, MNRAS, 430, 2446

Webb, J. K., King, J. A., Murphy, M. T., et al. 2011, PRL, 107, 191101

Xu, X., Padmanabhan, N., Eisenstein, D. J., Mehta, K. T., \& Cuesta, A. J. 2012, MNRAS, 427, 2146

Yang, T., Holanda, R., \& Hu, B. 2019, Astropart. Phys., 108, 57

1 Instituto de Física Téorica UAM-CSIC, Campus de Cantoblanco, 28049 Madrid, Spain

e-mail: matteo.martinelli@uam.es

2 Centro de Astrofísica da Universidade do Porto, Rua das Estrelas, 4150-762 Porto, Portugal

3 Instituto de Astrofísica e Ciências do Espaço, Universidade do Porto, CAUP, Rua das Estrelas, 4150-762 Porto, Portugal

4 Departamento de Física, FCFM, Universidad de Chile, Blanco Encalada 2008, Santiago, Chile
5 Institute of Space Sciences (ICE, CSIC), Campus UAB, Carrer de Can Magrans, s/n, 08193 Barcelona, Spain

${ }^{6}$ Institut d'Estudis Espacials de Catalunya (IEEC), 08034 Barcelona, Spain

7 School of Physics and Astronomy, University of Nottingham, University Park, Nottingham NG7 2RD, UK

8 INFN-Sezione di Torino, Via P. Giuria 1, 10125 Torino, Italy

9 Dipartimento di Fisica, Universitá degli Studi di Torino, Via P. Giuria 1, 10125 Torino, Italy

10 INAF-Osservatorio Astrofisico di Torino, Via Osservatorio 20, 10025 Pino Torinese (TO), Italy

11 INAF-IASF Milano, Via Alfonso Corti 12, 20133 Milano, Italy

12 AIM, CEA, CNRS, Université Paris-Saclay, Université Paris Diderot, Sorbonne Paris Cité, 91191, Gif-sur-Yvette, France

13 Université PSL, Observatoire de Paris, Sorbonne Université, CNRS, LERMA, 75014, Paris, France

14 CEICO, Institute of Physics of the Czech Academy of Sciences, Na Slovance 2, Praha 8, Czech Republic

15 Institut de Recherche en Astrophysique et Planétologie (IRAP), Université de Toulouse, CNRS, UPS, CNES, 14 Av. Edouard Belin, 31400 Toulouse, France

16 Université St Joseph; UR EGFEM, Faculty of Sciences, Beirut, Lebanon

17 Astrophysics Research Institute, Liverpool John Moores University, 146 Brownlow Hill, Liverpool L3 5RF, UK

18 INAF-Osservatorio di Astrofisica e Scienza dello Spazio di Bologna, Via Piero Gobetti 93/3, 40129 Bologna, Italy

19 INAF-Osservatorio Astronomico di Padova, Via dell'Osservatorio 5, 35122 Padova, Italy

20 Max Planck Institute for Extraterrestrial Physics, Giessenbachstr. 1, 85748 Garching, Germany

21 INFN-Sezione di Roma Tre, Via della Vasca Navale 84, 00146 Roma, Italy

22 Department of Mathematics and Physics, Roma Tre University, Via della Vasca Navale 84, 00146 Rome, Italy

23 INAF-Osservatorio Astronomico di Roma, Via Frascati 33, 00078 Monteporzio Catone, Italy

24 INAF-Osservatorio Astronomico di Capodimonte, Via Moiariello 16, 80131 Napoli, Italy

25 Institut de Física d'Altes Energies IFAE, 08193 Bellaterra, Barcelona, Spain

${ }^{26}$ Department of Physics "E. Pancini", University Federico II, Via Cinthia 6, 80126 Napoli, Italy

27 INFN section of Naples, Via Cinthia 6, 80126 Napoli, Italy

28 Centre National d'Etudes Spatiales, Toulouse, France

29 Institute for Astronomy, University of Edinburgh, Royal Observatory, Blackford Hill, Edinburgh EH9 3HJ, UK

${ }^{30}$ European Space Agency/ESRIN, Largo Galileo Galilei 1, 00044 Frascati, Roma, Italy

31 ESAC/ESA, Camino Bajo del Castillo, s/n, Urb. Villafranca del Castillo, 28692 Villanueva de la Cañada, Madrid, Spain

32 Department of Astronomy, University of Geneva, ch. d'Écogia 16, 1290 Versoix, Switzerland

${ }^{33}$ Univ Lyon, Univ Claude Bernard Lyon 1, CNRS/IN2P3, IP2I Lyon, UMR 5822, 69622 Villeurbanne, France

34 INAF-Osservatorio Astronomico di Trieste, Via G. B. Tiepolo 11, 34131 Trieste, Italy

35 Istituto Nazionale di Astrofisica (INAF) - Osservatorio di Astrofisica e Scienza dello Spazio (OAS), Via Gobetti 93/3, 40127 Bologna, Italy

36 Dipartimento di Fisica e Astronomia, Universitá di Bologna, Via Gobetti 93/2, 40129 Bologna, Italy

37 Istituto Nazionale di Fisica Nucleare, Sezione di Bologna, Via Irnerio 46, 40126 Bologna, Italy

38 Universitäts-Sternwarte München, Fakultät für Physik, LudwigMaximilians-Universität München, Scheinerstrasse 1, 81679 München, Germany 
39 Institute of Theoretical Astrophysics, University of Oslo, PO Box 1029, Blindern 0315, Oslo, Norway

40 Jet Propulsion Laboratory, California Institute of Technology, 4800 Oak Grove Drive, Pasadena, CA 91109, USA

41 von Hoerner \& Sulger GmbH, SchloßPlatz 8, 68723 Schwetzingen, Germany

42 Max-Planck-Institut für Astronomie, Königstuhl 17, 69117 Heidelberg, Germany

43 Aix-Marseille Univ, CNRS/IN2P3, CPPM, Marseille, France

${ }^{44}$ Mullard Space Science Laboratory, University College London, Holmbury St Mary, Dorking, Surrey RH5 6NT, UK

45 University of Lyon, UCB Lyon 1, CNRS/IN2P3, IUF, IP2I Lyon, France

${ }^{46}$ Université de Genève, Département de Physique Théorique and Centre for Astroparticle Physics, 24 quai Ernest-Ansermet, 1211 Genève 4, Switzerland

47 Department of Physics and Helsinki Institute of Physics, Gustaf Hällströmin katu 2, 00014 University of Helsinki, Finland

48 NOVA optical infrared instrumentation group at ASTRON, Oude Hoogeveensedijk 4, 7991PD Dwingeloo, The Netherlands

49 Argelander-Institut für Astronomie, Universität Bonn, Auf dem Hügel 71, 53121 Bonn, Germany

${ }^{50}$ Institute for Computational Cosmology, Department of Physics, Durham University, South Road, Durham DH1 3LE, UK

51 Université de Paris, 75013 Paris, France

52 LERMA, Observatoire de Paris, PSL Research University, CNRS, Sorbonne Université, 75014 Paris, France

53 Observatoire de Sauverny, Ecole Polytechnique Fédérale de Lausanne, 1290 Versoix, Switzerland

54 INFN-Sezione di Bologna, Viale Berti Pichat 6/2, 40127 Bologna, Italy

55 Institut de Física d'Altes Energies (IFAE), The Barcelona Institute of Science and Technology, Campus UAB, 08193 Bellaterra, Barcelona, Spain

56 Space Science Data Center, Italian Space Agency, Via del Politecnico snc, 00133 Roma, Italy

57 Institute of Space Science, Bucharest 077125, Romania

58 INFN-Padova, Via Marzolo 8, 35131 Padova, Italy

59 Dipartimento di Fisica e Astronomia "G.Galilei", Universitá di Padova, Via Marzolo 8, 35131 Padova, Italy

${ }^{60}$ Instituto de Astrofísica e Ciências do Espaço, Faculdade de Ciências, Universidade de Lisboa, Tapada da Ajuda, 1349-018 Lisboa, Portugal

61 Departamento de Física, Faculdade de Ciências, Universidade de Lisboa, Edifício C8, Campo Grande, 1749-016 Lisboa,

Portugal

62 Universidad Politécnica de Cartagena, Departamento de Electrónica y Tecnología de Computadoras, 30202 Cartagena, Spain

${ }^{63}$ Infrared Processing and Analysis Center, California Institute of Technology, Pasadena, CA 91125, USA

\section{Appendix A: The current BAO data}

Here we describe the currently available BAO data we use in our analysis. In particular, we use the measurements from 6dFGS (Beutler et al. 2011), SDDS (Anderson et al. 2014), BOSS CMASS (Xu et al. 2012), WiggleZ (Blake et al. 2012), MGS (Ross et al. 2015) and BOSS DR12 (Gil-Marín et al. 2016), DES (Abbott et al. 2019), Lya (Blomqvist et al. 2019), DR14 LRG (Bautista et al. 2018) and quasars (Ata et al. 2018).

The data provided by these surveys are described by the function $d_{z}$, defined in Sect. 4. The 6dFGs and WiggleZ BAO data are

\begin{tabular}{ccc}
$z$ & $d_{z}$ & $\sigma_{d_{z}}$ \\
\hline 0.106 & 0.336 & 0.015 \\
0.44 & 0.073 & 0.031 \\
0.6 & 0.0726 & 0.0164 \\
0.73 & 0.0592 & 0.0185
\end{tabular}

with their inverse covariance matrix given by

$C_{i j}^{-1}=\left(\begin{array}{cccc}4444.4 & 0 & 0 & 0 \\ 0 & 1040.3 & -807.5 & 336.8 \\ 0 & -807.5 & 3720.3 & -1551.9 \\ 0 & 336.8 & -1551.9 & 2914.9\end{array}\right)$,

and with the $\chi^{2}$ being

$\chi_{6 \mathrm{dFS}, \mathrm{Wig}}^{2}=V^{i} C_{i j}^{-1} V^{j}$,

and the data vector $V^{i}=d_{z, i}-d_{z}\left(z_{i}, \Omega_{\mathrm{m}, 0}\right)$.

The BAO measurements from MGS and SDSS (LOWZ and CMASS samples) are given by $D_{V} / r_{\mathrm{s}}=1 / d_{z}$ via

\begin{tabular}{ccc}
$z$ & $1 / d_{z}$ & $\sigma_{1 / d_{z}}$ \\
\hline 0.15 & 4.46567 & 0.168135 \\
0.32 & 8.62 & 0.15 \\
0.57 & 13.7 & 0.12
\end{tabular}

and the $\chi^{2}$ is then

$\chi_{\mathrm{MGS}, \mathrm{SDSS}}^{2}=\sum_{i}\left(\frac{1 / d_{z, i}-1 / d_{z}\left(z_{i}, \Omega_{\mathrm{m}, 0}\right)}{\sigma_{1 / d_{z, i}}}\right)^{2}$.

The BAO data from DES is of the form $d_{\mathrm{A}}(z) / r_{\mathrm{s}}$ with the data vector $\left(z, d_{\mathrm{A}}(z) / r_{\mathrm{s}}, \sigma\right)=(0.81,10.75,0.43)$ and the $\chi^{2}$ being

$\chi_{\mathrm{DES}}^{2}=\sum_{i}\left(\frac{d_{\mathrm{A}}(z, i) / r_{\mathrm{s}}-d_{\mathrm{A}}\left(z_{i}, \Omega_{\mathrm{m}, 0}\right) / r_{\mathrm{s}}}{\sigma_{d_{\mathrm{A}}(z, i) / r_{\mathrm{s}}}}\right)^{2}$.

The BAO data from Ly- $\alpha$ are of the form $f_{\mathrm{BAO}}=$ $\left((1+z) d_{\mathrm{A}} / r_{\mathrm{s}}, D_{\mathrm{H}} / r_{\mathrm{s}}\right)$ and are given by

\begin{tabular}{ccccc}
$z$ & $(1+z) d_{\mathrm{A}} / r_{\mathrm{s}}$ & $\sigma_{(1+z) d_{\mathrm{A}} / r_{\mathrm{s}}}$ & $D_{\mathrm{H}} / r_{\mathrm{S}}$ & $\sigma_{D_{\mathrm{H}} / r_{\mathrm{s}}}$ \\
\hline 2.35 & 36.3 & 1.8 & 9.2 & 0.36
\end{tabular}

with the $\chi^{2}$ being

$\chi_{\mathrm{Ly}-\alpha}^{2}=\sum_{i}\left(\frac{f_{\mathrm{BAO}, i}-f_{\mathrm{BAO}}\left(z_{i}, \Omega_{\mathrm{m}, 0}\right)}{\sigma_{f_{\mathrm{BAO}}}}\right)^{2}$.

The DR14 LRG and quasar BAO data assume $r_{\mathrm{s}, \mathrm{fid}}=147.78$ and are given by $D_{V} / r_{\mathrm{s}}=1 / d_{z}$

\begin{tabular}{ccc}
$z$ & $1 / d_{z}$ & $\sigma_{1 / d_{z}}$ \\
\hline 0.72 & $2353 / r_{\mathrm{s}, \text { fid }}$ & $62 / r_{\mathrm{s}, \text { fid }}$ \\
1.52 & $3843 / r_{\mathrm{s}, \text { fid }}$ & $147 / r_{\mathrm{s}, \text { fid }}$
\end{tabular}

and the $\chi^{2}$ being

$\chi_{\mathrm{LRG}, \mathrm{Q}}^{2}=\sum_{i}\left(\frac{1 / d_{z, i}-1 / d_{z}\left(z_{i}, \Omega_{\mathrm{m}, 0}\right)}{\sigma_{1 / d_{z, i}}}\right)^{2}$.

Finally, the total $\chi^{2}$ is

$\chi_{\mathrm{tot}}^{2}=\chi_{\mathrm{6dFS}, \mathrm{Wig}}^{2}+\chi_{\mathrm{MGS}, \mathrm{SDSS}}^{2}+\chi_{\mathrm{DES}}^{2}+\chi_{\mathrm{Ly}-\alpha}^{2}+\chi_{\mathrm{LRG}, \mathrm{Q}}^{2}$. 